\title{
Comparing the Calibration and Simulation Data of the Cryogenic Dark Matter Search
}

\author{
Anthony DiFranzo \\ Office of Science, Science Undergraduate Laboratory Internship (SULI) \\ Rensselaer Polytechnic Institute \\ SLAC National Accelerator Laboratory \\ Menlo Park, CA
}

August 20, 2010

Prepared in partial fulfillment of the requirements of the Office of Science, Department of Energy's Science Undergraduate Laboratory Internship under the direction of Rudy Resch at the Kavli Institute for Particle Astrophysics and Cosmology, Stanford Linear Accelerator Center.

Participant:

Signature

Research Advisor:

Signature 


\section{TABLE OF CONTENTS}

Abstract

$\begin{array}{ll}\text { Introduction } & 1\end{array}$

Materials and Methods $\quad 4$

$\begin{array}{ll}\text { Results } & 7\end{array}$

$\begin{array}{lr}\text { Discussion and Conclusions } & 8\end{array}$

$\begin{array}{lr}\text { Future Work } & 8\end{array}$

$\begin{array}{lr}\text { Acknowledgments } & 9\end{array}$

$\begin{array}{ll}\text { References } & 10\end{array}$ 


\begin{abstract}
Comparing the Calibration and Simulation Data of the Cryogenic Dark Matter Search. ANTHONY DIFRANZO (Rensselaer Polytechnic Institute, Troy, NY 12180) RUDY RESCH (Kavli Institute for Particle Astrophysics and Cosmology, Stanford Linear Accelerator Center, Stanford, CA 94025)
\end{abstract}

The Cryogenic Dark Matter Search, or CDMS, collaboration is preparing a new experiment called SuperCDMS. CDMS uses Germanium detectors to attempt the direct detection of dark matter. To do this, they measure the ionization and heat produced during an event where a WIMP scatters off of germanium crystal lattice. To prepare for the experiment the detectors are calibrated with various radioactive sources. The response of the detectors is also modeled by a Monte Carlo simulation. These simulations include modeling everything from the radiation production to the raw data collected by the detector. The experimental data will be used to validate the results of the detector simulation.

This research will look only at the phonons produced during events that occur very close to the detector surface. From the raw data and simulation output three parameters will be determined: the rise time, the decay time, and time to position independence. It was found that the simulation's risetime and time to position independence was generally smaller than that of the data, while the decay time was found to be larger in the simulation than in the data. These differences show that the simulation is not complete. The difference in risetime implies that the phonons are not spread out enough when they reach the detector walls, which would be improved by a look at the Luke phonon and charge transport. The long decay time in the simulation implies that the rate phonons are being absorbed is underestimated. Finally, the small time to position independence in the simulation could be due to a low phonon scattering rate. A simple solution may be to alter the parameters that control the simulation, while still remaining physically sensible, to help match simulation and data. 


\section{INTRODUCTION}

In 1934, Dark Matter was first hypothesized by Fritz Zwicky after approximating the mass of galaxies in the Coma Cluster. This average mass was two orders of magnitude greater than expected from the cluster's luminosity, he attributed the difference to what he called dark matter[1]. In 1959, Louise Volders discovered that the rotation curve of the spiral galaxy M33 did not match the predictions given by Newtonian Dynamics[2], see Figure 1 for a comparison of the expected and measured rotation curves. The two main hypotheses that resulted to explain these discoveries were the Modified Newtonian Dynamics, or MOND, and Dark Matter. MOND assumes that Newton's laws are incorrect at large distances, and the Dark Matter hypothesis says that there is invisible matter that is altering the gravitational field of a galaxy[3]. In 2006, a study was conducted on the Bullet Cluster, which consists of two galaxy cluster colliding. The visible matter, such as interstellar gas, was mapped using x-rays, while the total mass is mapped using gravitational lensing techniques, see Figure 2 for an image of this cluster. It was found that light was being bent where there was not very much visible matter, MOND is not able to explain this. It is believed that the collision separated the visible matter from the dark matter, which appears to have been formed in a halo around the galaxies.

Assuming Dark Matter exists, the first question one must ask is what is Dark Matter made of? It doesn't interact via the electromagnetic force, since we are not able to probe it with light. Dark Matter appears to be nearly non-interacting and predominantly nonrelativistic, often referred to as cold. Of the many candidates, Weakly Interacting Massive Particles or WIMPs are the most prominent. A WIMP is a hypothetical class of particles and that are believed to interact only through the weak force and gravity include the neutralino, Kaluza-Klein particle, and branon[4]. The discovery of neutralinos would be particularly exciting as it would give credibility to the Supersymmetric model[5].

Now the hypothesis must be tested; how will WIMPs be detected? In a collider, WIMPs, 
like neutrinos, must be found through the missing energy and momentum of a collision, as WIMPs will not interact well with detectors. Neutralinos are the most hoped for as constituents of dark matter. The neutralino is classified as a WIMP and is the supersymmetric partner of the neutrino, with an intrinsic spin of $3 / 2$ as determined by supersymmetry. The highest energies are currently attained with hadron, rather than lepton, colliders. Since hadrons are not elementary, the momenta of their constituents are not very well defined. Therefore, colliders can introduce some complications, however this is still a viable route. Indirect detection is also possible by looking for WIMP pair annihilation in space; however this is accompanied by other difficulties. The third option is direct detection which looks for the elastic scattering of WIMPs with the nuclei of a particle detector[5].

The Cryogenic Dark Matter Search, or CDMS, is an experiment looking to directly observe these particles and hopefully determine their candidacy as constituents of Dark Matter. The experiment consists of germanium detectors, which must be held at millikelvin temperatures, to detect an interaction between a dark matter particle and a Ge nucleus. Since dark matter is believed to be spread throughout our galaxy and is slowly moving we can move the detectors through space to attain collisions. As the Earth and our solar system move through the dark matter halo of the Milky Way Galaxy, the detectors will hopefully interact with a Dark Matter particle[5].

Data has been taken on the detector and a simulation has been developed to as closely as possible represent the conditions under which the data was taken. The purpose of the research discussed here is to compare the data with the simulation of the detector. This will either validate our model or tell us what needs to be improved or changed.

The detector geometry used in this experiment is known as an Interleaved Z-sensitive Ionization and Phonon, or iZIP. The top and bottom of the Ge detectors have contacts to collect charge and phonons. Figure 3 shows an image of the iZIP detector mask. These contacts are partitioned into regions, in order to gain information about the location of an interaction. Sensitivity to both the distribution and timing of phonon collection between 
each region determines where in the detector the event occurred. The phonon and charge channels allow us to determine the amount of energy in an interaction and are used to distinguish between nuclear and electron recoil, which is crucial in discriminating between signal and background. To further help with discrimination, these detectors are housed underground to reduce background events involving cosmic radiation.

Keeping the detectors at low temperatures has a dual purpose. First, a warm crystal lattice will naturally contain a high density of thermal phonons, which would swamp out any meaningful signal that might be present. Therefore, the detectors are held at millikelvin temperatures to minimize any presence of thermal phonons. Second, this low temperature is also required for our method of phonon detection. The phonon detection relies on Transition Edge Sensors, or TES, which need to be kept at the temperature where it transitions from a superconducting to normal state. Phonons are absorbed by the Quasiparticle-trap-assisted Electrothermal-feedback Transition-edge-sensor, or QET, a phonon will then break a cooper pair, which consists of two electrons bound together in a superconductor. The broken cooper pair releases quasiparticles which the QET is able to focus onto the TES at its center. The TES's heat up, bringing the sensor out of its superconducting state[6]. This transition is accompanied by a sharp, measurable increase in the resistance of the sensor, which is recorded as part of our data collection.

There are three forms of phonons in our detector that we must study to properly analyze the data. The first are called Luke phonons, these are radiated from charged particles that move greater than the speed of sound within that medium. This is analogous to Cherenkov radiation, where a charged particle radiates light when it moves faster than the speed of light in that medium. The second types are called intrinsic phonons, which are released at the position of the interaction. They usually undergo anharmonic decay, where a phonon will split into two phonons each with about half the energy of the parent phonon. The mean free path of this type of decay scales inversely to the phonon energy. Phonons with low enough energy will have a mean free path larger than the size of the detector. At this 
point, the phonons are called ballistic; they bounce around the detector without scattering until it is absorbed. The third are known as recombination phonons[5]. These are released when electrons and holes recombine, often through a defect states rather than a direct transition across the band gap, known as Shockley Reed Hall recombination. The defect state population is usually greater at the edges of a crystal lattice; therefore most recombination phonons are created at the surfaces of the detector.

Charge transport will also be integral to modeling our detectors. An electric field is applied across the detector to draw electrons and holes to opposite sides of the detector. However, an important process to account for is oblique propagation. Here electrons will not travel in a straight line following the electric field. Instead, they will follow "energy valleys" within the germanium, this makes them travel at an angle to the electric field. However, the energy valleys for the holes are aligned with the electric field lines, so they do not experience this angled transportation described here.

\section{MATERIALS AND METHODS}

The Monte Carlo simulation of the events serves as a way for us to prove we understand the detector. There are three distinct components to this simulation: radiation scattering, transport, and measurement. In the first step GEANT4 places the source near to one face of the detector, masks can be placed between the source and detector in the program to better replicate the experimental setup. The incident particles are then allowed to scatter within the Ge. GEANT4 simulates the positions and energies of these scattering events. In the second step, these positions and energies are used to determine the initial number and locations of the phonons and charges. These phonons and charges propagate through the detector and undergo the transport processes previously discussed. Finally, when they reach the surface the phonons are collected. For simplicity in the simulation, the detector faces are divided into regions. The number of phonons incident on each region are counted and 
put through a simulation of the QET system. The final result is a pulse that models the output of the actual detector during a calibration data run.

In order to validate the simulation, we can compare to a calibration dataset on the detectors to which we will compare the simulation. The detectors are calibrated with radioactive sources such as Barium-133, Californium-252, and Cadmium-109. Each of these calibration sources provide a different type of event that we are interested in. Ba-133 produces relatively high energy electron and gamma rays, this will produce bulk electron recoils. Cf-252 produces neutrons which will produce nuclear recoils. Cd-109 produces lower energy electron and gamma rays, which produce electromagnetic events that occur very close to the surface of our detector[7]. We are specifically interested in surface events for this project, meaning Cd-109 will be used as the source for our data analysis. To ensure that the simulation and data are actually measuring the same thing, we can help ensure this by applying quality cuts to our data that remove events that do not match what the simulation is attempting to model. For example, we may be only looking at a certain energy range or perhaps we want to isolate surface events.

Both the data and simulation output raw pulses. These raw pulses are current measured through each channel, which is related to the number of phonons being absorbed. Figure 4 shows an example of such a data pulse. After some time phonons have diffused uniformly throughout the detector. At this point phonons must slowly leak out, leading to a long decay tail in the pulse that will be seen in all eight of the channels. The lower plot shows these same pulses but are all scaled so that the slow decay tails match up. Note that Figure 1 contains a diagram of the channel regions: one is located on the bottom and the other is located on the top of the detector. Also, note that the regions on top are orthogonal to the regions on the bottom which gives us more information on the position of events. See Figure 5 for a diagram of the channel geometry.

The data and simulation pulses are then analyzed. The preliminary analysis includes determining, for each event, the decay time, the rise time, the amount of energy deposited 
into each channel, etc. These parameters are then plotted in a histogram so data and simulation can be directly compared. The histograms are normalized so that all their areas are unity. Observing differences at this level serves as a gauge of how well we understand our detector and can help determine the most important input parameters for the simulation.

I will focus on three parameters. The first is called the $10-40 \%$ Rise Time. This is the time between the points when the phonon pulse is 10 and $40 \%$ of its maximum height. This piece of data gives us information of phonon transportation, particularly the Luke phonons since they reach the detector surface first. This also serves to discriminate between event types. Surface events vary in recoil energy and ionization energy, therefore they can easily be misinterpreted as nuclear or electron recoil events. However, surface events are also accompanied by fast rise times, while bulk recoils tend to have slow rise times. Surface events tend to associate with faster risetimes because phonons produced close to the surface may not have a chance to down convert before being absorbed into the QET. This means that high energy phonons are being absorbed. These phonons are able to break several cooper pairs instead of one, which in turn produces a faster rise time.

The second parameter is the decay time. This is the time it takes for the pulse to decay to a factor of $e$ of its maximum height; this is a typical convention for any given exponential decay. The intrinsic phonons undergo anharmonic decay and eventually become ballistic. In this mode they are unlikely to down convert and will travel more readily to a surface. Therefore, the phonon pulse will decay faster as more and more phonons enter the ballistic regime. The decay time then allows us to see how long it takes before phonons become ballistic and if the down conversion process is properly modeled. This also tells us if our absorption rate into the QET is correct.

The third is known as the time to position independence. This is the amount of time before we can no longer tell where an event occurred by looking at the pulses. In other words, at this time phonons have diffused uniformly throughout the detector. This again will tell us if the phonon transport processes have been modeled properly. The main factor 
influencing position independence is the down conversion rate, that is the rate at which phonons undergo anharmonic decay.

The aforementioned simulation must account for many processes, including those related to transportation in the crystal. Other basic considerations comprise probability of phonon absorption into the QET and how much energy will be collected into each partition in the sensor. The main goal of this research is to construct a complete analysis and comparison between simulation and calibration data sets. We are mainly concerned with data sets involving Cd-109, which as previously stated produces surface events.

\section{RESULTS}

The following are all based on phonon output from Cd-109 simulation and data runs. The 10-40\% Rise Time Histogram is shown in Figure 6. The top plots the rise time for the fastest channel for each event, note that this may not be the same channel for every event. The bottom plots the slowest risetime for each event. The data is plotted in gray and the simulation in black dotted lines. In the data, the slowest channel has a mean of $6 \mu$ s and a standard deviation of $3 \mu \mathrm{s}$, while in the simulation gives a mean of $4 \mu \mathrm{s}$ with a standard deviation of $2 \mu \mathrm{s}$. The fastest channel has a mean of $54 \mu \mathrm{s}$ in the data with a standard deviation of $12 \mu \mathrm{s}$. For fastest channel in the simulation the mean is $48 \mu \mathrm{s}$ and a standard deviation of $8 \mu \mathrm{s}$. Note that these values are approximate.

The Decay Time histogram is shown in Figure 7. The differences are very apparent here, as the data distribution is broader and its mean is smaller than that of simulation. The data appears to decay about $200 \mu$ s faster than the simulation. However, they do share the same functional form: both have very clean Gaussian distributions. Also, note the height difference between the two distributions, this is due to normalization.

The Time to Position Independence Histogram is shown in Figure 8. The simulation shows position independence being reached around $250 \mu \mathrm{s}$, while data shows it to most 
likely occur around $400 \mu \mathrm{s}$. Additionally, note that their functional forms match as they are both Gaussian with longer tails to the right.

\section{DISCUSSION AND CONCLUSIONS}

For both the fastest and slowest risetimes the simulation appears to be slightly faster than the data. This could imply that the simulation Luke phonons reach the detectors too late, in that they are measured too close in time with phonons that should be arriving later. This effect should be sensitive to anharmonic decay since we are looking at surface events, meaning the decay rate may be too high. Another contributing factor will be our model of charge transportation, since it is the motion of the charges that produces these Luke phonons.

The simulation predicts a longer decay time than measured, which could indicate a problem with the QET model. If the absorption rate into the QET is underestimated, then phonons will remain in the detector for a longer period of time. The long decay time could also be due to the rate intrinsic phonons enter the ballistic regime. If the simulation underestimates the rate at which phonons down convert, it will also take longer for the pulse to decay.

The simulation predicts the events to reach position independence sooner than measured. This could mean that simulation phonons are down converting at too high of a rate. A high down converting rate will result in more athermal phonons, so they should statistically distribute themselves faster, which means the detector will reach position independence sooner.

\section{FUTURE WORK}

The differences in the three parameters seem to be consistent. The absorption into the QET's and the rate of anharmonic decay seem to be common themes. It is not completely 
clear as to how the anharmonic decay rate needs to be changed, though it appears to be too high. The next step is to tune the parameters used in the simulation to see if a good match can be made. If this fails then we will need to return to the physics processes involved and see what can be improved or included to better match what is physically occurring.

Currently, a probability is assigned to determine if a phonon is absorbed when it reaches a QET. A model of the physics occurring at the detector surface could be implemented to form a more physically realistic representation. The charge model could also be further developed; this should improve agreement in the rise times between data and simulation. Currently, the phonon pulse output is the majority of the analysis. If the simulation for measuring charge were improved it could also be used in the analysis, and could at least help our understanding of Luke phonon production. Further analysis needs to be done to determine what is actually causing the differences between data and simulation.

The SLAC CDMS group now has more data and simulation analyzed. The program to do these comparisons is now fully functioning, therefore other data sets can be analyzed with minimal changes to the program code. Germanium is also not well understood at very low temperatures, so the data taken will help us to determine what changes to make to our model. Outside of the comparison, the simulation can also be used to test different detector geometries before they are made. Though this method is not perfect, it will be very useful to exclude certain geometries before the trouble is gone through to fabricate the detectors.

\section{ACKNOWLEDGMENTS}

This work was conducted at SLAC National Accelerator Laboratory and was funded by the United States Department of Energy, through the SULI program. I would like to thank my mentor, Rudy Resch, for his guidance and taking the time to explain what I didn't understand. I would also like to thank Dr. Eduardo do Couto e Silva, for checking in on my progress and moving my project along near stumbling blocks. The whole CDMS 
collaboration at SLAC has been very helpful and supportive of my project; I want to thank all of them. The SULI staff, who worked to make this program run smoothly, provided great lectures for us, and ensured we had a productive summer. Finally, I want to thank all of my fellow SULI students who made this summer exciting and more than just about research.

\section{REFERENCES}

[1] F. Zwicky, "On the Masses of Nebulae and of Clusters of Nebulae," Astrophysical Journal, vol. 86, p. 217, October 1937.

[2] L. Volders, "Neutral Hydrogren in M 33 and M 101," Bulletin of the Astronomical Institutes of the Netherlands, vol. 14, pp. 323-334, 1959.

[3] R. Sanders and E. Noorfermeer, "Confrontation of MOND with the Rotation Curves of Early-type Disc Galaxies," Mon.Not.Roy.Astron.Soc, p. 9, February 2008.

[4] C. N. Bailey, "The Cryogenic Dark Matter Search: First 5-Tower Data and Improved Understanding of Ionization Collection," Ph.D. dissertation, Case Western Reserve University, Cleveland, January 2010. [Online]. Available: http: //cdms.berkeley.edu/Dissertations/cnbailey_thesis.pdf

[5] J. Filippini, "A Search for WIMP Dark Matter Using the First Five-Tower Run of the Cryogenic Dark Matter Search," Ph.D. dissertation, University of California, Berkeley, Berkeley, December 2008. [Online]. Available: http: //cdms.berkeley.edu/Dissertations/filippini.pdf

[6] S. Leman, "Development of Phonon-Mediated Transition-Edge-Sensor X-ray Detectors for Use in Astronomy," Ph.D. dissertation, Stanford University, Palo Alto, September 2006. [Online]. Available: http://proquest.umi.com/pqdlink? vinst=PROD\&attempt= $1 \& \mathrm{fmt}=6 \&$ startpage $=-1 \&$ ver $=1 \&$ vname $=\mathrm{PQD} \& \mathrm{RQT}=309 \& \operatorname{did}=1221714061 \& \exp =$ 08-08-2015\&scaling =FULL\&vtype $=$ PQD\&rqt=309\&TS=1281375994\&clientId=83448 
[7] Z. Ahmed et al., "Analysis of the Low-Energy Electron-Recoil Spectrum of the CDMS Experiment," Physical Review D, vol. 81, p. 4, July 2009. 


\section{FIGURES}

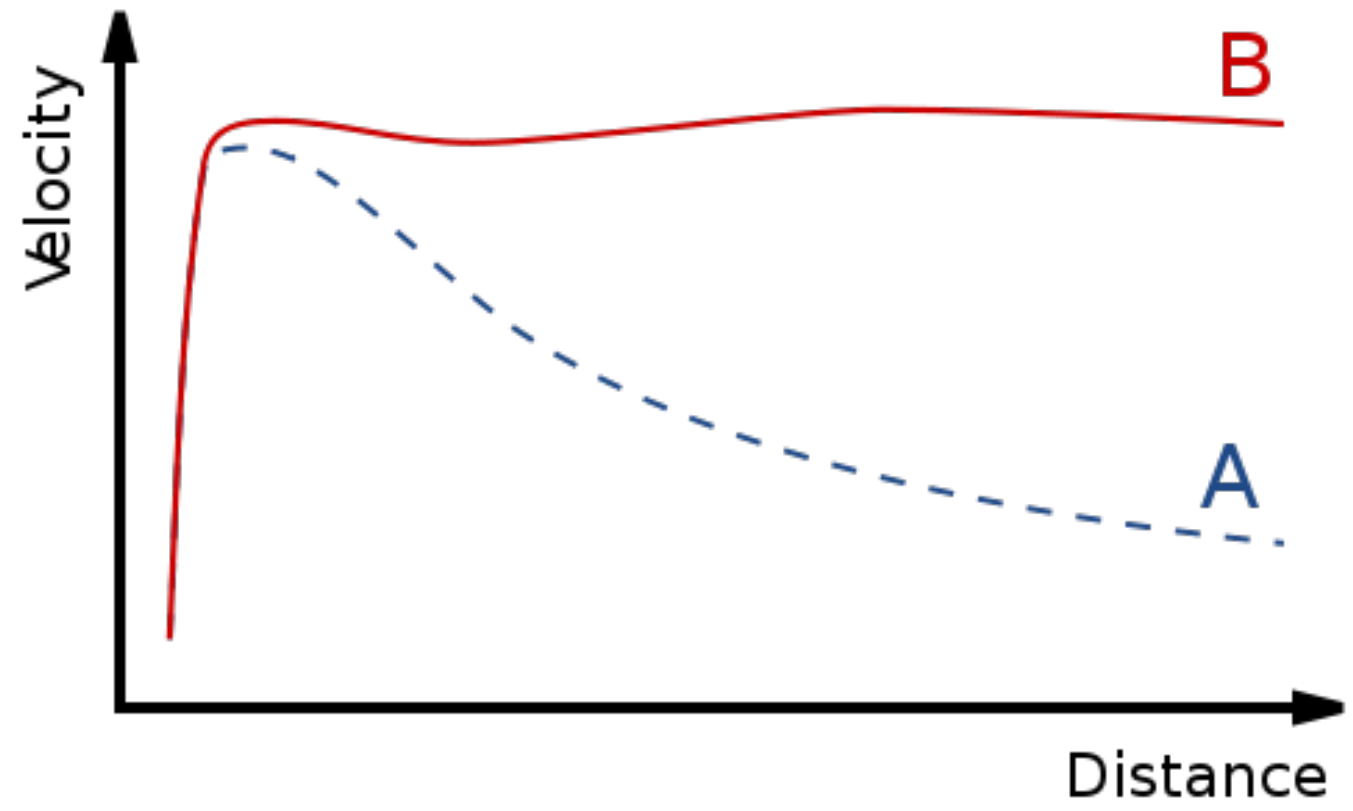

Figure 1: An example of a galaxy curve, note that distance is measured radially from the center of a galaxy. With Newtonian Dynamics we expect curve A, however we observe curve B. 


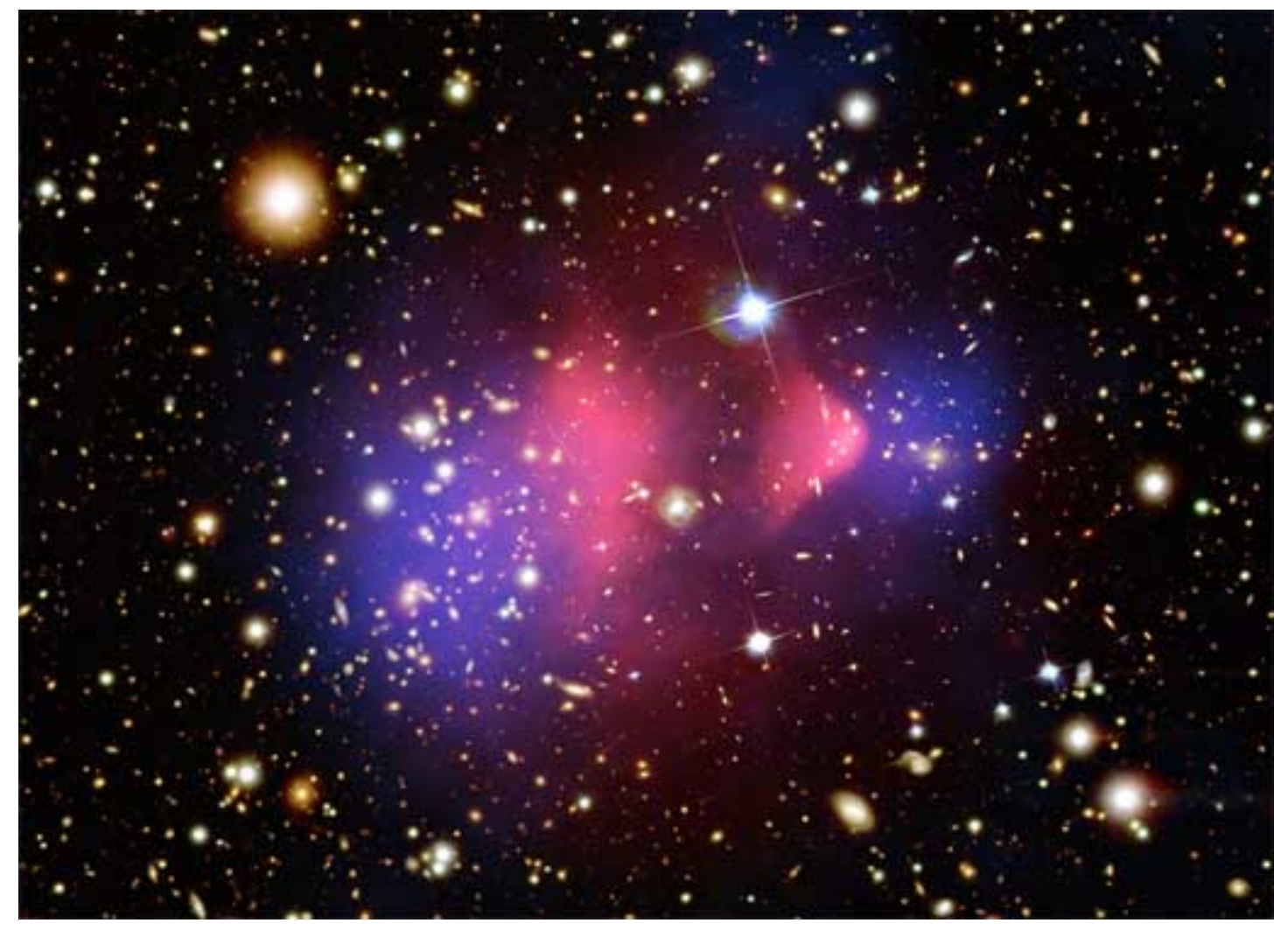

Figure 2: This is an x-ray and gravitational map of the Bullet Cluster. The pink at the center represents the x-ray image of the interstellar gas, while the blue is the dark matter that been separated from the rest of the cluster. The dark matter here was mapped using gravitational lensing. 


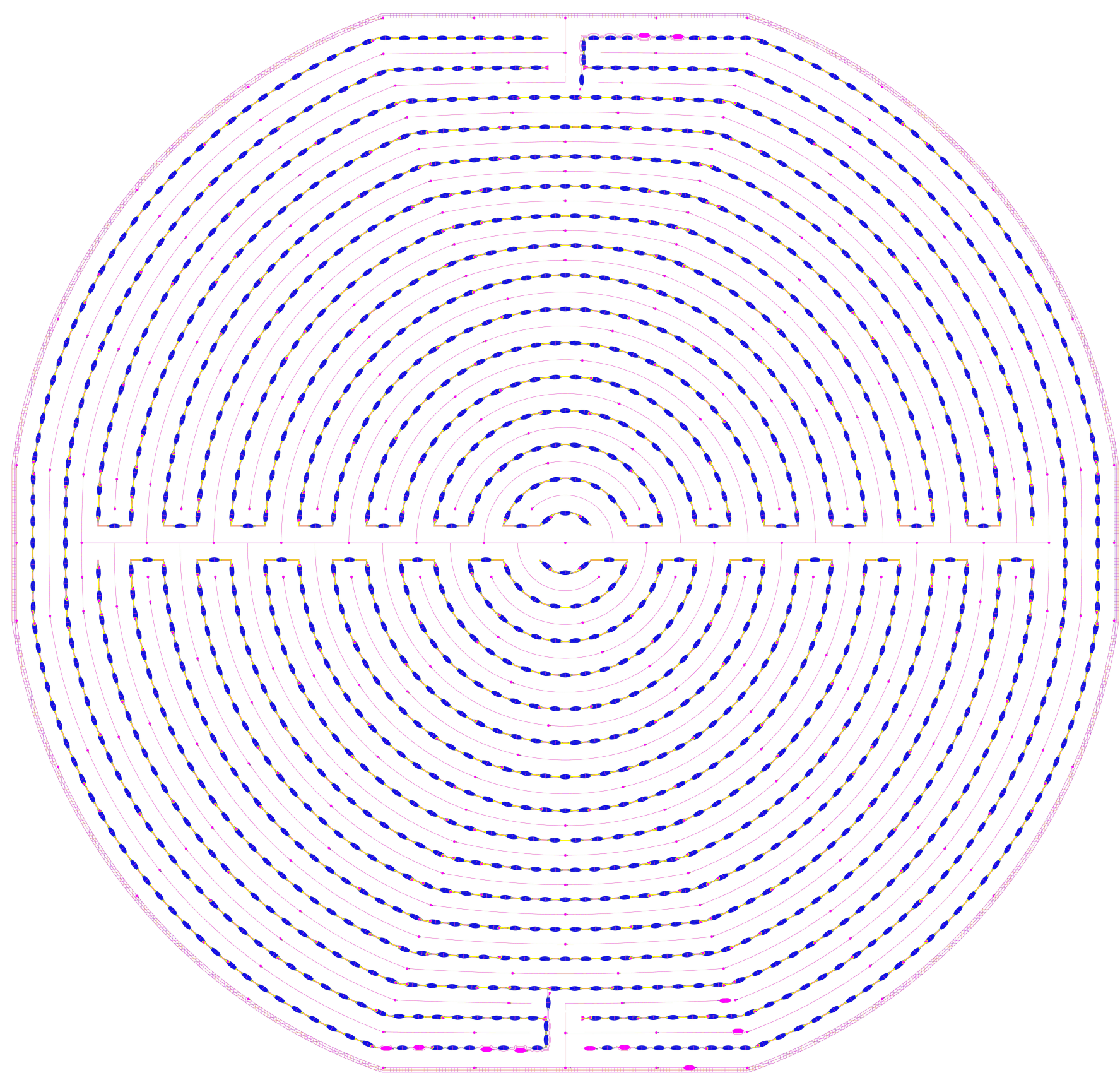

Figure 3: This is an image of the sensor geometry placed on the top of the detector. The blue sensors are the QET's, which contain the TES's. The pink sensors collect the charge. There are four separate sets of QET's on this side, all QET's that are connected are part of the same channel. 

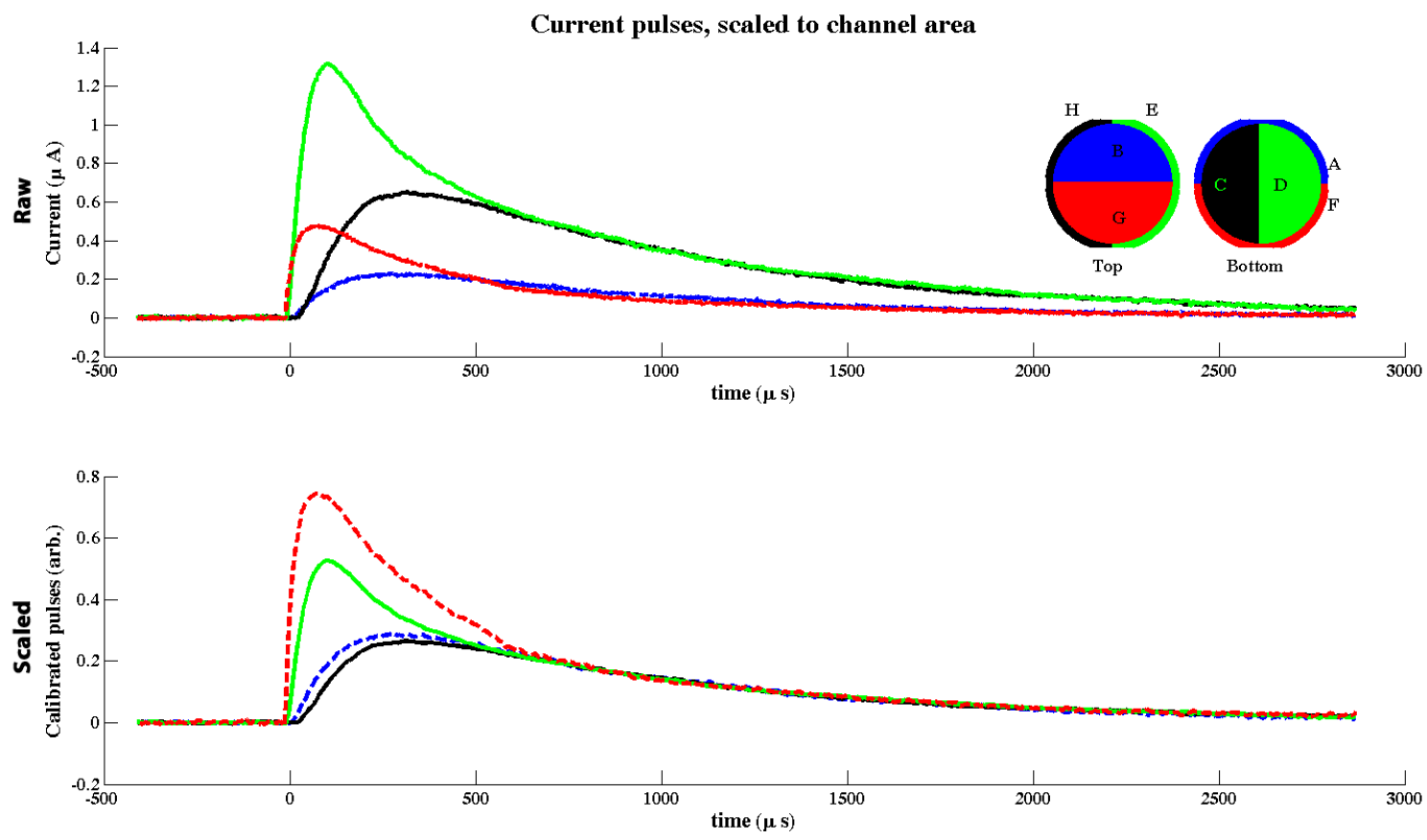

Figure 4: A typical plot of the phonon pulse. The top contains the raw data pulses, the bottom pulses are scaled. A diagram of the sensor regions on the detectors are located in the upper right corner. Note that only the bottom four channels have been plotted for simplicity. The dotted lines denotes data from an outer channel.

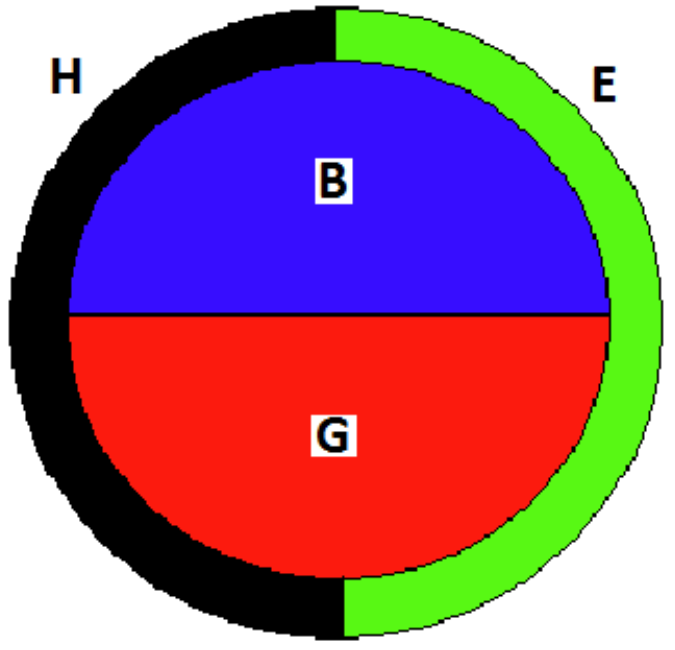

Top

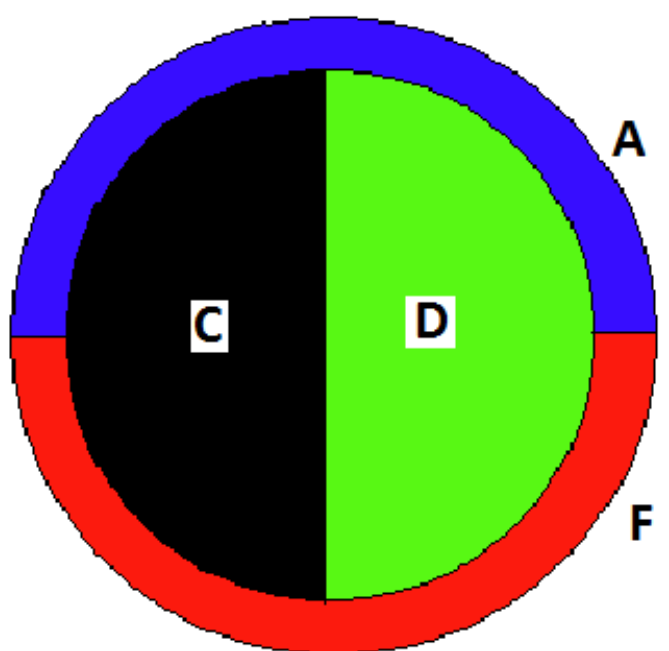

Bottom

Figure 5: A diagram of the eight channels, which are located on the top and bottom of the detector. Channels labeled A, E, F, and $\mathrm{H}$ are called outer channels, while those labeled B, $\mathrm{C}, \mathrm{D}$, and $\mathrm{G}$ are known as inner channels. The top and bottom surfaces are oriented so that their geometries are orthogonal. A separate pulse is attained from each of the eight. 

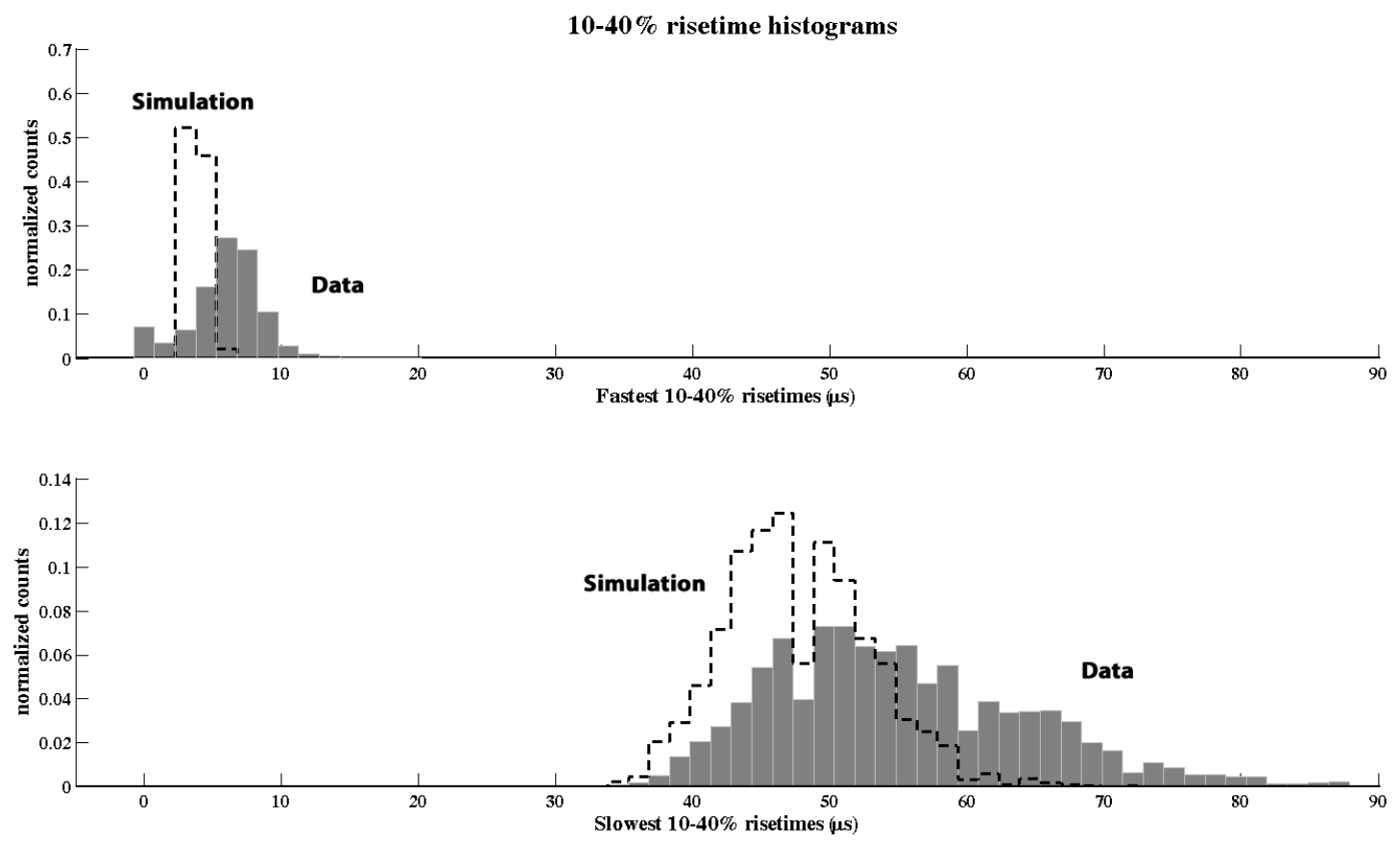

Figure 6: A plot of the slowest(top) and fastest(bottom) risetime for each event. Data is in gray and simulation is outlined in black dotted lines. The data for each has a similar range and distribution.

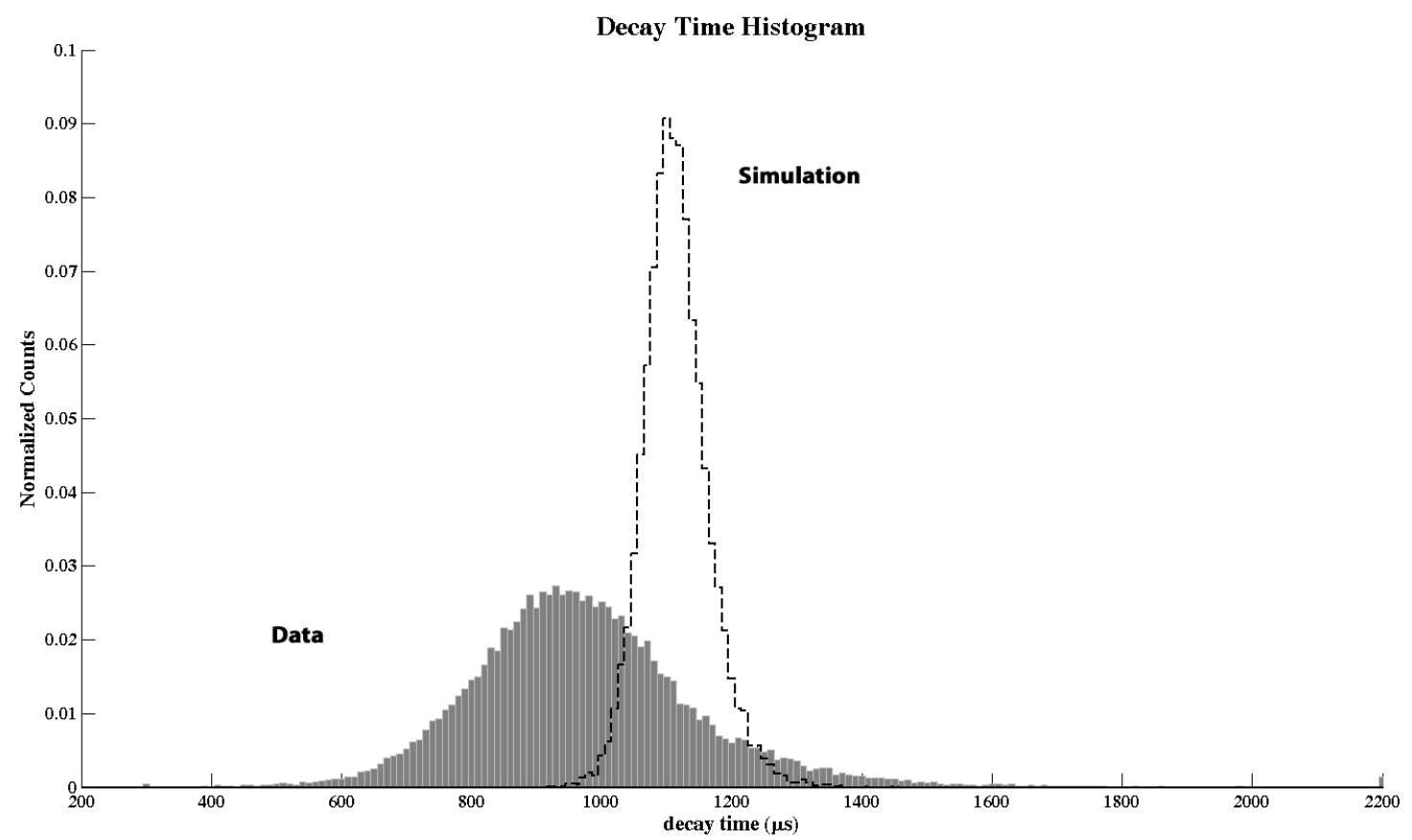

Figure 7: The distribution of the decay times for data and simulation. The simulation has a longer decay time and a thiner distribution, however both share a Gaussian functional form. 


\section{Time to position independent signals}

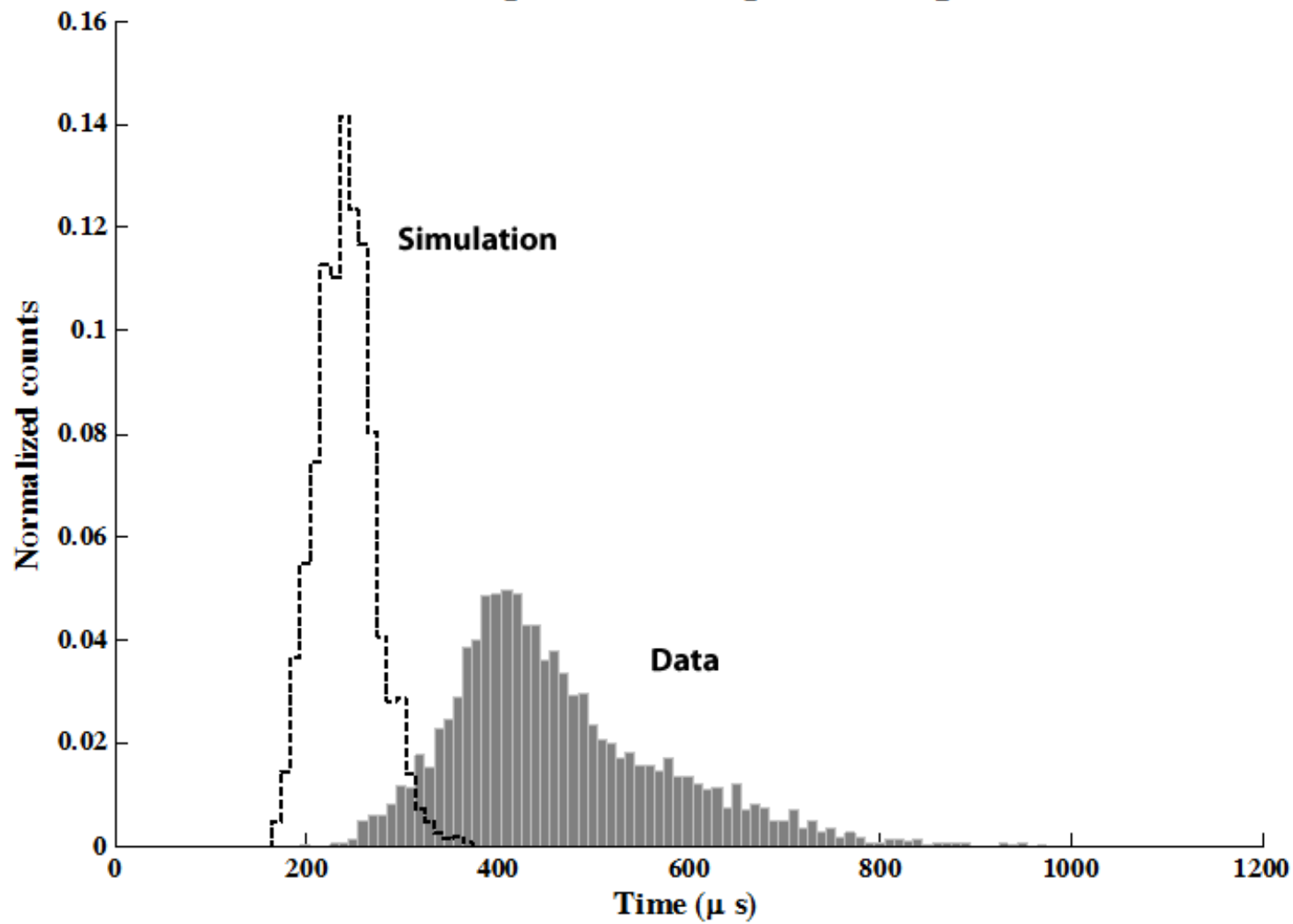

Figure 8: The time to position independence distributions. Note that they both have longer tails to the right. 